\title{
BMJ Open Quality Development and implementation of electronic medical handovers across psychiatric hospitals: quality improvement initiative
}

\author{
Luke Skelton $\left(1,,{ }^{1}\right.$ Jonathan Rogers, ${ }^{2}$ Chris Kalafatis ${ }^{3}$
}

To cite: Skelton L, Rogers J, Kalafatis C. Development and implementation of electronic medical handovers across psychiatric hospitals: quality improvement initiative. BMJ Open Quality 2019;8:e000630. doi:10.1136/ bmjoq-2019-000630

Received 8 January 2019 Revised 31 October 2019 Accepted 1 November 2019
Check for updates

(C) Author(s) (or their employer(s)) 2019. Re-use permitted under CC BY-NC. No commercial re-use. See rights and permissions. Published by BMJ.

1John Dickson Ward, South London and Maudsley NHS Foundation Trust, London, UK ${ }^{2}$ Specialty Registrar, South London and Maudsley NHS Foundation Trust \&Wellcome Trust Clinical PhD Fellow, University College London, London, UK

${ }^{3}$ Clinical Trials, South London and Maudsley NHS Foundation Trust, London, UK

Correspondence to Dr Luke Skelton; luke.skelton@slam.nhs.uk

\section{ABSTRACT}

A lack of integration between internal processes and failure to use the full potential of information technology (IT) systems is common in psychiatric hospitals. We aimed to reduce the number of out-of-hours medical errors by ensuring that there is consistent and transparent weekend medical handover by creating an electronic handover system that is easy to use, robust and embedded into the existing trust IT systems. We employed quality improvement (QI) methodology to address this issue. After trialling in a single site followed by six cycles of improvement, the weekend medical handover system is now in use across four boroughs and has been integrated into trust policy. It has received qualitative and quantitative evidence of improvement, with $100 \%$ of doctors reporting the system improved patient care and a 64\% (from 11 to 4 events/year) reduction in moderate, severe and catastrophic adverse incidents occurring out-of-hours within the older adult service $\left(p=0.29, \chi^{2} 1.117\right)$.

The increasing number of complex patients with comorbid medical illness in psychiatric inpatient services demands robust handover systems similar to that of an acute trust. This Ql work offers a template for achieving this across other psychiatric trusts and demonstrates the positive change that can be achieved.

\section{WHY WAS CHANGE NEEDED?}

Across South London and the Maudsley NHS Trust (SLaM), the inpatient medical teams were handing over patients to the out-ofhours medical cover on an informal basis. These handover processes were not standardised, and there was significant variability across different sites and staff. The handover was largely verbal and either relied on the doctor's memory or individual paper notes that were often lost or misplaced. Being a large, London-based mental health trust with many specialty services, SLaM is busy trust while also providing 24 hours' care to a complex patient cohort.

The weekend out-of-hours cover at SLaM consists of three doctors covering long days (Friday, Saturday and Sunday) and one doctor covering the nights, meaning that patient information handed over could pass through up to three people before reaching the intended doctor. The effects of this were that mistakes were being made out-ofhours whereby jobs were being missed, and there was no consistent and transparent way of ensuring safe medical handover. Consequently, the ward teams reported that handed-over jobs were not completed or not documented by the out-of-hours team. This is a breach of clinical governance guidance and compromises patient safety. This was particularly relevant for the older adult wards within the trust, where the complexity of medical comorbidity among this patient group often required a greater level of intervention over the weekend.

Deficiencies in the medical handover and out-of-hours medical provision had been found to contribute to two significant events occurring on an older adult ward, which in part had driven the change. The overarching aim of this piece of work was to reduce the number of out-of-hours mistakes by ensuring there is a consistent and transparent weekend medical handover and, in doing so, to improve the medical team's experience of out-of-hours processes. This will be achieved via two clear objectives: to create a trust-wide electronic medical handover list that can be edited and documented within the electronic note system, Patient Journey System (ePJS), and to create a handover system that is effective, easy to use and is the same across all boroughs within the trust.

\section{BACKGROUND}

Failure to handover effectively has long been recognised as a major preventable cause of patient harm, yet there continues to be large variability of handover processes across NHS trusts. ${ }^{1}$ A large-scale European Commission project found that poor handover communication is responsible for $25 \%-40 \%$ of adverse events. $^{2}$ Despite guidance on safe clinical 
handover having been around for many years, individual hospitals employ different systems leading to a lack of standardisation and contributing to varying degrees of effectiveness. ${ }^{3}$ Hospital medicine requires round-theclock care, provided by multiple clinicians working shift patterns. The need for consistency and continuity, despite the changing staff, points towards a structured and standardised handover process.

Research into electronic handover within medical and surgical specialties has found that a standardised proforma approach relaying patient information improves compliance with and clarity of handover processes, and that predefined fields rather than free text space are helpful. ${ }^{45}$ It has previously been shown that logistical difficulties in accessing computer terminals can be a limitation of electronic handovers. ${ }^{6}$

Psychiatric hospitals are perceived to experience less clinical demand out-of-hours, and perhaps this is why they have often lagged behind the acute trust setting in developing formal handover initiatives. ${ }^{7-9}$ However, due to the increasing complexity of psychiatric inpatients, the physical and mental health comorbid burden, and the drive towards providing a 24-hour service, there is a need to implement the same robust clinical handover practices within inpatient psychiatric settings. ${ }^{10-12}$ This is most evident on older adult psychiatric inpatient wards where the case mix is similar to that of an acute geriatric ward with a comparable out-of-hours clinical demand. ${ }^{13-16}$

\section{DATA COLLECTION}

Initial questionnaires were administered to gauge the needs of the target population and to gather data on the trainees' views on the adequacy of the current out-ofhours handover procedures. After implementation of the electronic handover, further questionnaires were administered to the trainee body in order to produce qualitative and quantitative data of the staff's perception of the new medical handover process.

\section{Retrospective data collection}

Data on adverse incidents are collected via Datix reports within the trust of SLaM. These are a compulsory record of incidents that can be completed by any member of the multidisciplinary team. In a ward environment, these are more often completed by nursing staff. The reports include a nature of incidents, including medication errors, violent behaviour, acute medical deterioration or transfers to an acute trust.

In order to gather quantitative data on the impact of the electronic weekend handover, data on adverse incidents were compared from a 1-year period before implementation and a 1-year period after implementation. Due to the scope of this review, only the older adults service was reviewed. This service was chosen as this piece of quality improvement (QI) work was born out of this service due to the complexity of this patient group.
All of the Datixed incidents occurring within the older adult services across the trust were reviewed for the time periods of 1 January 2016-31 December 2016 (preimplementation) and 1 July 2017-30 June 2018 (postimplementation). All of the incidents that occurred on the inpatient wards and were either moderate, severe or catastrophic in severity were reviewed in the clinical notes to assess whether the incident was related to out-ofhours care delivery. If an incident occurred out-of-hours or during the working day of Monday, the clinical notes were reviewed for medical input out-of-hours. The clinical notes were then reviewed to establish whether the patients' condition had been noted to be deteriorating during the in-hours period preceding the incident. Datix reports had been completed by nursing staff and were therefore independent of and not influenced by the new handover process.

\section{DESIGN}

After considering different forms of electronic handover of medical information, the project team met with the information technology (IT) team responsible for managing the ePJS, the electronic notes system used within SLaM. After two face-to-face meetings and email liaison, we were able to build a handover function into the existing electronic notes. The out-of-hours medical team would have access to an out-of-hour list, which contained any patients whom the inpatient ward medical teams felt needed review out-of-hours. The ward teams documented the handover material (job details, background medical summary and need to know information) in a progress note, and ePJS would autopopulate key demographics (name, date of birth, ward location, mental health act status and trust ID). As the electronic weekend handover was built into ePJS, it was live and editable, and so the out-of-hours medical team could click on the list and be taken directly to the person's notes, where they can then document any outcomes or results of the review. This also served the function that the medical team for Sunday would be able to know whether the patient had been reviewed by the medical team on Saturday, allowing for more effective prioritisation. At this stage of development, the handover focused on the out-of-hours period from Friday, 17:00, through Monday, 09:00.

\section{STRATEGY}

In setting out to achieve the aim of this project, a driver diagram was created to graphically represent the theory of change and plan QI activities (figure 1). A PlanDo-Study-Act (PDSA) QI model was implemented throughout the project. The QI project core team was made up of two CT1 psychiatry trainees, with the support of an older adult psychiatry consultant. IT support was provided a centralised ePJS team, and trust management had input when integrating the new handover process into trust protocol. 


\section{The development and implementation of electronic medical handovers across}

\section{psychiatric hospitals: A Quality Improvement initiative}

AIM

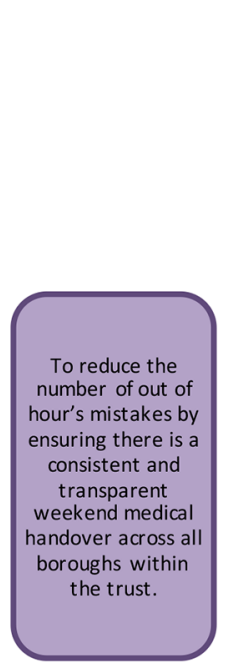

PRIMARY DRIVERS

SECONDARY DRIVERS

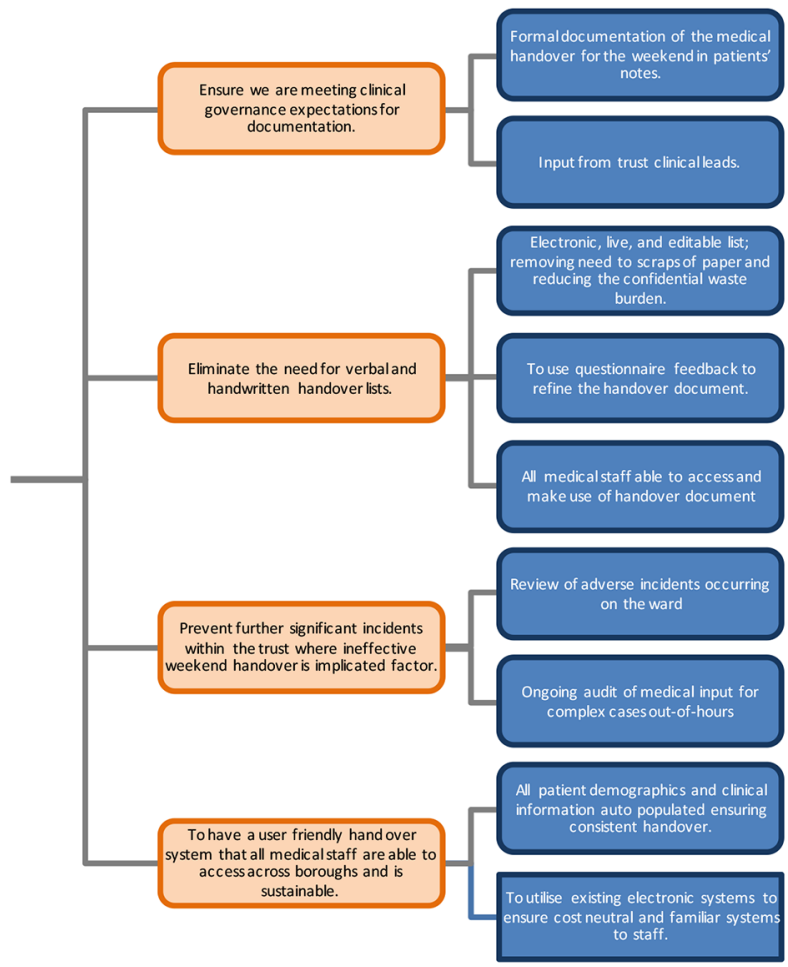

CHANGE IDEAS

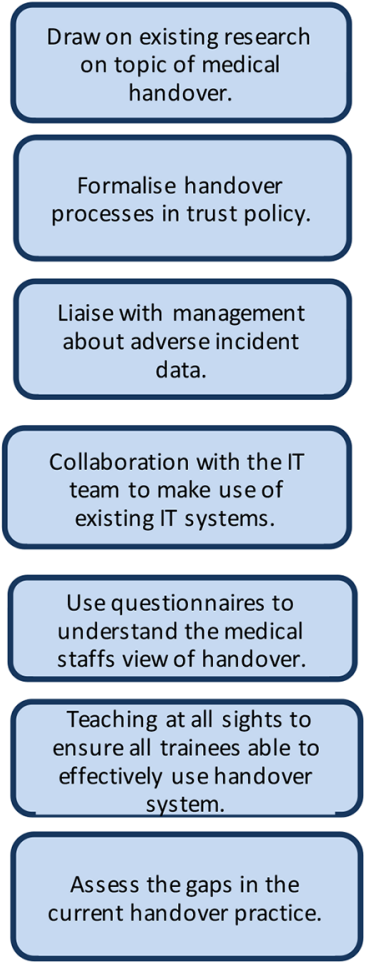

Figure 1 Driver diagram for the project outlining the aim, primary and secondary drivers, and the changes implemented to achieve these. IT, information technology.

First, as the changes were primarily going to be affecting the day-to-day practice of the core trainees within the trust, a questionnaire was administered to gauge the needs of the target population. The formal feedback was reviewed and used to further guide the driver diagram. In conjunction with the ePJS support team, a function was designed within the existing IT system which could allow for a live and editable handover of jobs and autofilling of all other necessary demographics. Using 'tester' patient profiles, we trialled and changed different versions of the handover list until a function was arrived at, whereby the 'progress note' entry into the notes was pulled through into the 'Weekend Medical Handover' report on ePJS. The relevant patient demographics autopopulated this report, ensuring there was accurate information for the out-of-hours team. A user guide was created as both a word document and video file, and this was disseminated to all trainees via email and, in addition, hard copies were distributed in the on-call rooms. The project was trialled at local level (on one hospital site) for 2 weeks alongside the existing handover process. The first few PDSA cycles identified and fine-tuned how the demographic data and core handover data would be visualised in the handover report; at this stage, the IT team was closely involved. Several PDSA cycles were then completed as feedback was provided by the trainees using the new electronic system; key issues that were resolved included ease of access to the handover within the electronic notes and ensuring access for locum staff working in the trust.

Lecture slides were created and these were presented at each of the four hospital sites' local teaching days, with questions being fielded during these sessions. These were 30 min face-to-face sessions to supplement instruction manuals sent via email.

A further meeting with the IT team occurred to produce an individual weekend medical handover report for each of the boroughs, which captured data entered from any of the inpatient wards within that hospital site. Again, this required the use of 'tester patients' to ensure that all patient data intended for weekend handover were collated in the correct site's report. As per the initial trial of the project, trainees were instructed to use the existing handover processes in parallel to the new weekend medical handover for the first week to ensure 
any problems could be addressed safely; following this, a transition to the new handover process was undertaken. The teaching materials were later incorporated into the local induction sessions for each of the hospital sites within SLaM and were included in the induction pack, which human resources provide to locums working in the trust.

\section{RESULTS}

\section{Questionnaire analysis}

There were 101 full-time doctors consisting of both general practice trainees and core psychiatry trainees who undertook out-of-hours duties across the four sites within SLaM and therefore participated in the handover process. The questionnaire was administered via trust email, and there were 24 responses out of a possible 101 giving a questionnaire response rate of $24 \%$.

Of the 24 doctors who responded to the questionnaires, $96 \%$ felt that the information they receive during handover was inadequate to safely assess the patient. There were $67 \%$ of doctors who felt that handover information had been lost prior to being seen by the out-ofhours doctor. Notably, $100 \%$ of doctors felt that handover processes could be improved.

One of the primary drivers of the project was to have a user-friendly handover system that all medical staff can access across the four boroughs. Of those that responded formally, $83 \%(20 / 24)$ of the trainee body found the weekend medical handover application easy to use. In addition to this, there was a $100 \%$ uptake in trainees using the new handover process after the introductory period, which reinforces that on the whole, the new handover system was user friendly.

One hundred percent of the questionnaire responders felt that the weekend medical handover application improved patient care. In addition to the quantitative data collected by the questionnaires, there was white space for responders to offer comments they may have about the handover.

One responder wrote:

'No information gets lost. It is very slick and easy to use. My initial fear was that jobs would not be filtered creating extra work load. However, this has not happened during my on calls.'

A second responder wrote:

'Forces people to evaluate why they are handing over a patient to be reviewed. With the demands of a weekend handover we cannot have a lot of 'just in case' reviews. The reviews need to be task orientated, with an appropriate focus on plan from the team.'

A third responder wrote

'It's great that all the patient information is automatically generated, I worry about relying on IT systems.'
Table 1 Adverse incidents occurring within the older adult inpatient population preintervention and postintervention

Preimplementation Postimplementation 1 January 2016-31 1 July 2017-30 June December 2016 2018

\begin{tabular}{lcc}
$\begin{array}{l}\text { Incidents in- } \\
\text { hours* }\end{array}$ & 10 & 8 \\
$\begin{array}{l}\text { Incidents out- } \\
\text { of-hourst }\end{array}$ & 11 & 4 \\
Total & 21 & 12 \\
\hline
\end{tabular}

*In-hours incidents represent those occurring within the normal working week.

†Out-of-hours incidents detailed here refer to those occurring between 17:00, Friday, and 09:00, Monday, that is, the weekend period.

The first two above comments corroborate that another of the primary drivers was achieved, eliminating the need for inadequately detailed, verbal and handwritten handover lists. The use of an entirely electronic handover process means that there is no longer a verbal handover of each patient requiring input from the out-of-hours team over the weekend. The fact that the handed-over information and out-of-hours intervention remain in the patients' electronic notes beyond the weekend ensures that we are meeting the clinical governance expectations, therefore achieving another primary driver.

\section{Adverse incident analysis}

All incidents occurring on the older adult inpatient wards were reviewed for the year leading up to the implementation of the new system (1 January 2016-31 December 2016). There were 21 incidents that met the criteria for a review of the clinical notes; of these, 11 occurred outof-hours and could have benefitted from a more robust handover process (table 1). In the observed period postimplementation (1 July 2017-30 June 2018), there were 12 incidents that met the criteria for a review of the clinical notes; of these, 4 occurred out-of-hours and could have benefitted from a more robust handover (table 1). When comparing the preimplementation and postimplementation time periods, there was an overall reduction of $43 \%$ (from 21 to 12 events/year) of moderate, severe and catastrophic incidents across the older adult inpatient setting. Furthermore, there was a 64\% (from 11 to 4 events/year) reduction in the number of incidents occurring where out-of-hours handover processes could have played a role. This did not reach statistical significance $\left(p=0.29, \chi^{2} 1.117\right)$ but showed an important trend, given the rarity of moderate, severe or catastrophic adverse incidents. There was a small reduction in the number of events occurring in-hours (from 10 to 8 events/year), which, given how infrequently these events occur and the lengthy measurement period (1 year), likely represents a chance finding. The reduction in out-of-hours events was 3.5-fold greater over the same measurement period. 
It is worth noting that for the adverse incidents recorded postimplementation, 2 of the 12 incidents were acute, 'out of the blue' events, and after the acute event had been managed, the electronic weekend handover was used to more closely monitor these individuals out-of-hours.

\section{Soft measures}

In addition to the formal questionnaire feedback and quantitative data gathered from adverse incidents, there are a number of soft measures that evidence the success of the electronic weekend medical handover. First, one of the drivers for this piece of QI work was that there were frequent verbal and written complaints from the medical teams on the inpatient wards that jobs were not being completed or documented by the out-of-hours staff. Following implementation of the new handover process, these complaints appear to have diminished, and it has been supported by both trainees and consultants within SLaM. The electronic weekend medical handover was reviewed by the senior clinicians within the trust and has now been written into the SLaM out-of-hours policy and can be found in the South London and the Maudlsey Out-of-Hours Policy 2017 document.

\section{DISCUSSION}

This piece of work is an example of how QI methodology can be applied to drive positive change to local systems and then how this can act as a blueprint for regional level change. As well as achieving the overarching aim of reducing the number of out-of-hours mistakes and thereby improving patient care, this project has led to streamlining of the handover processes across multiple sites. The integration of existing IT systems to meet the clinical needs of out-of-hours medical provision meant that this was a cost-neutral development and has led to implementation of uniform handover processes across the four inpatient sites within SLaM. Although the reduction in adverse incidents did not reach statistical significance, moderate, severe and catastrophic incidents are rare, low incidence events; therefore, clinicians view even one event as too many. There is no evidence to suggest direct causality; however, the QI aimed to address at least one potential contributing factor to out-of-hours incidents, and the trend in data supports that this was achieved. Second, this was received very positively by clinicians as it also addresses continuity of care, simplifies admin work, creates an audit trail and helps to monitor out-of-hours care delivery.

Within NHS services more generally, but particularly within psychiatry, there is an underuse of the existing and expensive IT systems. ${ }^{17}$ There is a lack of shared knowledge and learning between those tasked with providing clinical care and those who manage the IT systems. This can lead to a lack of cohesion and underusage of the technology already available within NHS trusts. This project has highlighted the need to move towards maximising the function of IT systems to reduce errors, improve documentation and make the workload of an overstretched service provider a little bit more succinct. The integrated medical handover approach adopted as part of this project was shown to be effective in a 2013 review by Flemming and Hübner. ${ }^{18}$ The review showed that by integrating electronic handovers into existing electronic patient records, errors could be reduced and outcomes improved. In addition, using the same handover processes across the four sites within the trust reduces the burden placed on human resources and streamlines the process of inducting new doctors into the trust, again reducing room for error.

Due to the overall reduction in mental health bed numbers, psychiatric trusts have seen an increased acuity of the case mix present on inpatient psychiatric wards. ${ }^{19}$ Among the older adult's population, there is a complex picture of comorbidity and often concurrent physical health problems not dissimilar to patients found on geriatric acute medical wards. ${ }^{14}$ Both of these factors indicate that there has to be a shift in the way in which we provide out-of-hours medical care to ensure that the clinical needs of these patients are met over the weekends and between 17:00 and 09:00. Where there has previously been a reactive approach to medical provision out-of-hours, this new formal handover process marks a shift towards proactive clinical care for patients' out-of-hours, consequently preventing unnecessary admissions to the acute trust. The wealth of relevant information integrated into the electronic handover and the improved continuity from the 09:00 to 17:00 medical cover into that provided outof-hours is a step in the direction of consistent 24 hours' care.

Although it is not possible to imply causality, the reduction in adverse incidents occurring within the older adults' inpatient services since the implementation of electronic handover could indicate that it had a preventative role. This would mirror the views of the doctors using the handover, $100 \%$ of whom felt that patient care was improved. The findings of this piece of work suggest that formalising and integrating out-of-hours handover processes into the electronic patient records reduce errors, increase clarity and lead to better patient care. In turn, a similar approach should be considered by other mental health trusts nationally, as their electronic notes allow for easy and seamless adaptation.

\section{Limitations of this project}

As in many survey-based projects, the questionnaire response rate achieved was lower than preferred, but the white-space text added richness to the responses. Due to the time and personnel constraints, the review of adverse incident rates within the trust had to be limited to the older adults' service, and therefore, these data may not be generalisable to the general adult population. That said, this patient group often have the more complex mix of physical and psychiatric comorbidities.

As the adverse incidents of interest were relatively infrequent, which meant a full year was observed 
preintervention and postintervention, due to this large time interval, it was not possible to make three separate measurements, which would have been preferred when attributing change secondary to a QI initiative.

The adverse incidents were identified by one reviewer, and there was no assessment of the reliability of these judgements.

\section{CONCLUSION}

This was a small pilot project in a single site, which is now fully functioning across four boroughs and has been written into SLaM out-of-hours policy with continued successful use. The findings of this piece of work suggest that formalising and integrating out-of-hours handover processes into the electronic patient records can help reduce errors, increase clarity and lead to better patient care. There is a need to look inwardly as well as outwardly in order to ensure we are maximising the potential of the existing systems. In order to meet the needs of an increasingly acute and complex case mix, care provision needs to shift towards an acute medical model of out-ofhours cover. This piece of work could act as a template for other psychiatric trusts facing a similar set of problems and could be readily adapted for the variety of electronic systems in use.

Contributors LS and JR designed and conducted the study with overview of the project by CK. LS wrote and submitted the article with review and input from both JR and CK.

Funding The authors have not declared a specific grant for this research from any funding agency in the public, commercial or not-for-profit sectors.

Competing interests None declared.

Patient consent for publication Not required.

Provenance and peer review Not commissioned; externally peer reviewed.

Open access This is an open access article distributed in accordance with the Creative Commons Attribution Non Commercial (CC BY-NC 4.0) license, which permits others to distribute, remix, adapt, build upon this work non-commercially, and license their derivative works on different terms, provided the original work is properly cited, appropriate credit is given, any changes made indicated, and the use is non-commercial. See: http://creativecommons.org/licenses/by-nc/4.0/.

ORCID iD

Luke Skelton http://orcid.org/0000-0002-1643-9582

\section{REFERENCES}

1 Royal College of Physicians. Acute care toolkit 1: handover. London: RCP, 2011. https://www.rcplondon.ac.uk/guidelines-policy/acutecare-toolkit-1-handover

2 Barach P. Handover: improving the continuity of patient care through identification and implementation of novel patient handover processes in Europe. Project website, 2015. Available: http://www. handover.eu/index.html [Accessed 28 Oct 2018].

3 British Medical Association. Safe handover: safe patients. Guidance on clinical handover for clinicians and managers. London: BMA, 2004. https://www.bma.org.uk/-/media/Files/./safe\%20handover\% 20safe\%20patients.pdf

4 Till A, Sall H, Wilkinson J. Safe handover : safe patients - the electronic handover system. BMJ Qual Improv Rep 2013;2:u202926. w1359.

5 Cheah L-P, Amott DH, Pollard J, et al. Electronic medical handover: towards safer medical care. Med J Aust 2005;183:369-72.

6 Govier M, Medcalf P. Living for the weekend: electronic documentation improves patient handover. Clin Med 2012;12:124-7.

7 Raptis DA, Fernandes C, Chua W, et al. Electronic software significantly improves quality of handover in a London teaching hospital. Health Informatics J 2009;15:191-8.

8 Cleary M, Walter G, Horsfall J. Handover in psychiatric settings: is change needed? J Psychosoc Nurs Ment Health Serv 2009;47:28-33.

9 Pullen I, Loudon J. Improving standards in clinical record-keeping. Advances in Psychiatric Treatment 2006;12:280-6.

10 Thornicroft G. Physical health disparities and mental illness: the scandal of premature mortality. Br J Psychiatry 2011;199:441-2.

11 Leucht S, Burkard T, Henderson J, et al. Physical illness and schizophrenia: a review of the literature. Acta Psychiatr Scand 2007;116:317-33.

12 Saxena S, Thornicroft G, Knapp M, et al. Resources for mental health: scarcity, inequity, and inefficiency. Lancet 2007;370:878-89.

13 Bradshaw LE, Goldberg SE, Lewis SA, et al. Six-Month outcomes following an emergency hospital admission for older adults with comorbid mental health problems indicate complexity of care needs. Age Ageing 2013;42:582-8.

14 Bartels SJ. Caring for the whole person: integrated health care for older adults with severe mental illness and medical comorbidity. $J$ Am Geriatr Soc 2004;52:S249-57.

15 Seow LSE, Chong SA, Wang P, et al. Metabolic syndrome and cardiovascular risk among institutionalized patients with schizophrenia receiving long term tertiary care. Compr Psychiatry 2017;74:196-203.

16 Zubenko GS, Marino LJ, Sweet RA, et al. Medical comorbidity in elderly psychiatric inpatients. Biol Psychiatry 1997;41:724-36.

17 Hillestad R, Bigelow J, Bower A, et al. Can electronic medical record systems transform health care? potential health benefits, savings, and costs. Health Aff 2005;24:1103-17.

18 Flemming $D$, Hübner $U$. How to improve change of shift handovers and collaborative grounding and what role does the electronic patient record system play? Results of a systematic literature review. Int J Med Inform 2013;82:580-92.

19 Care Quality Commission. The state of care in mental health services 2014 to 2017, 2017. Available: https://www.cqc.org.uk/ sites/default/files/20170720_stateofmh_report.pdf [Accessed 3 Oct 2018]. 